$15^{\text {th }}$ International Conference on

AEROSPACE SCIENCES \& AVIATION TECHNOLOGY,

$\boldsymbol{A S A T}$ - 15 - May 28 - 30, 2013, Email: asat@mtc.edu.eg,

Military Technical College, Kobry Elkobbah, Cairo, Egypt,

Tel: +(202) 24025292 -24036138, Fax: +(202) 22621908

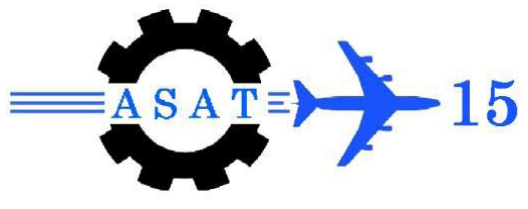

\title{
Performance Enhancement of Low Cost Non-GPS Aided INS for Unmanned Applications
}

\author{
\{M. M. Abbas, A. M. Kamel, Y.Z. El-Halwagy, R. A. Albordany ${ }^{*}$
}

\begin{abstract}
Low cost Autonomous Navigation Systems (ANS) used in Unmanned Arial Vehicles (UAVs) usually depend on Global Navigation Satellite Systems (GNSS) as a primary mean of navigation. For military applications, it is not accepted to use GNSS as a sole mean for navigation as it is susceptible to jamming and signal interference. For that reason, integrated navigation systems that compromise GNSS and other sensors such as Inertial Navigation System (INS) are used. INS as a standalone navigation system suffers from a huge number of errors which makes it impossible to use the low cost and grade ones for long term navigation unless aided by other means such as magnetometers.
\end{abstract}

This paper describes the INS, its basic principles, and types. The test results of low cost NonGPS aided INS for unmanned applications that use commercial off-the-shelf Micro-ElectroMechanical System (MEMS) accelerometers, gyroscopes, and magnetometers are introduced. An inertial measurement unit (IMU), different types of gyroscopes, accelerometers, and magnetometer theory of operation are illustrated. Real time attitude and drift computations are performed using MEMS IMU and magnetometer. A test result comparison between IMU and magnetometer is executed for attitude measurements. Magnetometer calibration is achieved for accurate attitude measurements. A Kalman filter is also used to smooth the output data for IMU and magnetometers. Test results showed that attitude measurements using integrated gyroscopes outputs suffer from high drift rate in roll, pitch, and yaw. Using accelerometers aiding through a complementary filter, roll and pitch drift are compensated. Using magnetometers aiding, yaw (heading) is also compensated. Test results show that the sensors used in the combo MEMS IMU board under test suffer from high drift in yaw which reaches about 11 degrees after 5 minutes. Using a well calibrated magnetometer aiding, the error in yaw is nearly compensated.

Keywords: Inertial navigation system, micro-electro-mechanical system, inertial measurement unit, magnetometers, Kalman filter.

\section{Nomenclature}
$\phi, \theta, \psi$
roll, pitch and yaw Eulerangles
$\dot{\phi}, \dot{\theta}, \dot{\psi}$
roll, pitch and yaw Euler angle rates
$P, Q, R$
Body roll, pitch and yaw angular rate
$\phi_{m}, \theta_{m}$
Measured roll and pitch angles by IMU
$a_{x}, a_{y}, a_{z}$
Vehicle acceleration components measured by IMU

Egyptian Armed Forces, Egypt. 


\section{Introduction}

Navigation is the process of knowing the position, velocity, and direction. Inertial navigation is accomplished by integrating the output of a set of sensors to compute position, velocity, and attitude. These sensors are gyroscopes which measure angular rate with respect to inertial space and accelerometers which measure linear acceleration. The triad that contains 3 gyroscopes and 3 accelerometers is called Inertial Measuring Unit (IMU). If navigation processing is performed on the IMU outputs the system is upgraded to an Inertial Navigation System (INS). Although integration is considered a simple process, but complexities arise due to the various coordinate frames encountered, sensor errors, and noise in the system. Due to these errors, depending on low cost inertial navigation sensors to obtain navigation parameters is very hard unless aided by other navigation sensors. Nowadays Global Navigations Satellite Systems (GNSS) and particularly the American Global Positioning System (GPS) is the most widespread navigation system. In certain applications, GPS is not a possible solution. In certain areas, e.g. in mountainous surroundings, the GPS signal is either not present or very inaccurate because of multipath interference. In addition to that, GPS signal is very week and easy to be jammed, intentionally interfered, or even can be taken over through a spoofing operation. Furthermore, navigation using GPS is dependent on the satellites governed by the USA which might not always be accessible during a war situation. Lastly the precision using GPS may be very poor, and not suitable for precision navigation needed by e.g. a missile or a war ship [1].

A lot of researches have been done to enhance the performance of the INS navigation by integrating it with GPS through a various number of algorithms. Indeed, these researches are very valuable and already applied to a lot of navigation modules available in the market but almost all of them deal with a tactical grade inertial sensors and assuming GPS signal is guaranteed with low possibility of jamming or spoofing. For this reason, this research is focused on dealing with low cost IMUs available in the market and to process their data to obtain attitude information which is the heart and source of error for the whole navigation process. To enhance the performance of low cost IMUs, the research is directed to use other GNSS sensors for the above reasons. Among other GNSS sensors comes the magnetometer which senses the earth magnetic field and hence direction information in the azimuth plane can be obtained. The choice of magnetometers was because its availability, and - if well calibrated- accuracy.

The rest of this paper is divided as follows:

1. Section (2) is an introduction to the INS.

2. Section (3) is about attitude computing using the chosen IMU.

3. Section (4) is about magnetometers, sources of errors, and calibration methods.

4. Section (5) introduces the enhancement method used for attitude optimal estimation.

5. Section (6) is the summary and conclusions.

\section{Inertial Navigation System (INS)}

Strap down inertial navigation consists of three major parts: Alignment, navigation and aiding. During the alignment phase, the alignment algorithm estimates the initial value of the body frame with respect to the navigation frame, that is, the roll, pitch and yaw of the system. This is used by the navigation algorithm to transform the measurements from the body frame to the navigation frame. By requiring the IMU to be stationary during alignment, this can be done by measuring gravity and earth rotation. When the alignment phase has obtained a sufficiently accurate estimate of the roll, pitch and yaw, the navigation phase can begin. During navigation, a navigation algorithm continuously calculate the roll, pitch and yaw from 
inputs from the gyroscopes, so acceleration measurements can be resolved in the navigation frame and integrated to yield velocity and subsequently positions with respect to the earth. Ultimately, this results in the latitude, longitude, height, velocity and attitude outputs, which are the primary variables of interest in inertial navigation systems. However, because of sensor errors and the double integration from acceleration to position, inertial navigation systems tend to drift exponentially from the true values. This shortcoming can be overcome by aiding the INS with various external sensors which can bind the errors propagating in the INS algorithms. An aiding algorithm uses knowledge about the errors in the INS and the aiding device to "fuse" the information from the two systems in an optimal way. A good example is GPS aiding, which can bound the position and velocity of the "fused" INS/GPS system whilst keeping the precision of the INS.

The sensors used in INS all have errors, some of which are reproducible and hence correctable. Before inertial sensors are incorporated into an INS, these errors are identified by a calibration algorithm [1].

\subsection{Basic Principle of Inertial Navigation}

Given the ability to measure the acceleration of vehicle it would be possible to calculate the change in velocity and position by performing successive mathematical integrations of the acceleration with respect to time. In order to navigate with respect to our inertial reference frame, it is necessary to keep track of the direction in which the accelerometers are pointing. Rotational motion of the body with respect to inertial reference frame may be sensed using gyroscopic sensors that are used to determine the orientation of the accelerometers at all times. Given this information it is possible to resolve the accelerations into the reference frame before the integration process takes place. An inertial navigation uses gyroscopes and accelerometers to maintain an estimate of the position, velocity, and attitude rates of the vehicle in or on which the INS is carried, which could be a land vehicle, aircraft, spacecraft, missile, surface ship, or submarine. The combination of an IMU and a computer running navigation equations is called an Inertial Navigation System (INS) as shown in Fig. 1, [2].

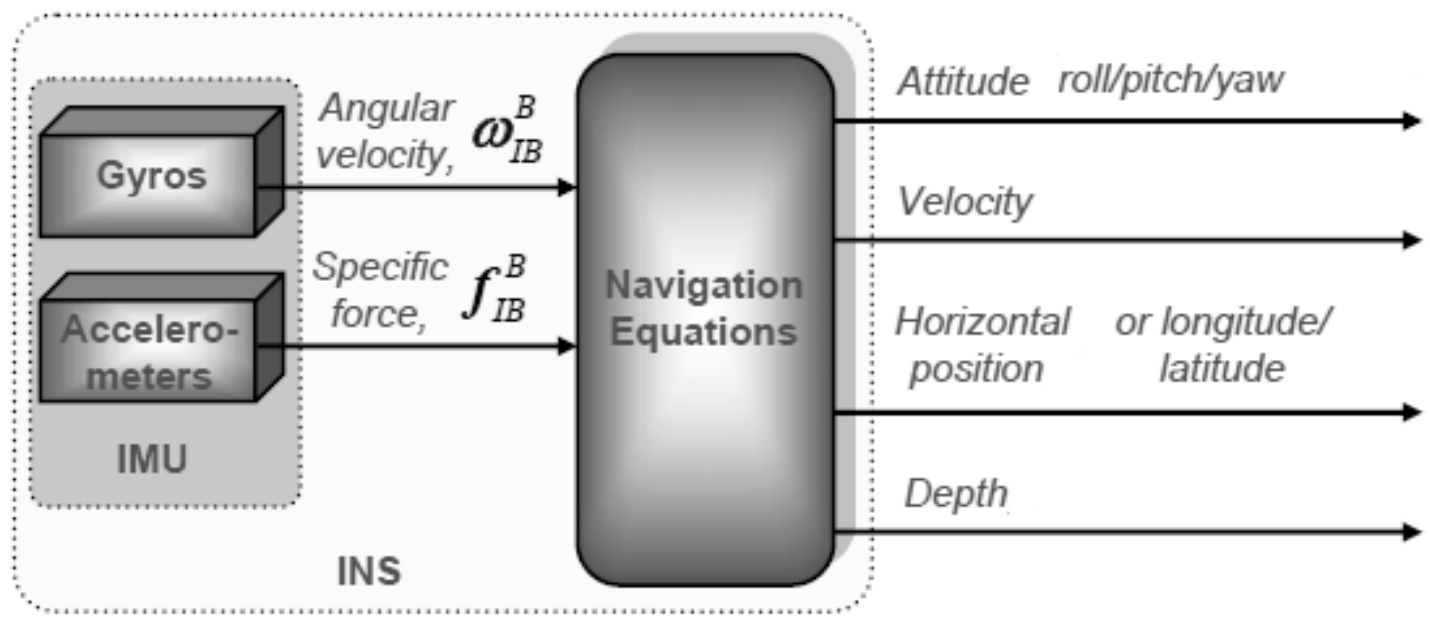

Fig. 1 Inertial navigation system simplified block diagram

\subsection{Aided Inertial Navigation System}

All inertial navigation systems suffer from integration drift: small errors in the measurement of acceleration and angular velocity are integrated into progressively larger errors in velocity, which are compounded into still greater errors in position. Since the new position is 
calculated from the previous calculated position and the measured acceleration and angular velocity, these errors accumulate roughly proportionally to the time since the initial position was input. Therefore the position must be periodically corrected by input from some other type of navigation system. To limit the drift, an INS is usually aided by other sensors like GPS and compasses (magnetometers) that provide direct measurements of for example position and velocity. The different measurements are blended in an optimal manner by means of a Kalman filter. An aided INS block diagram is shown in Fig. 2, [3].

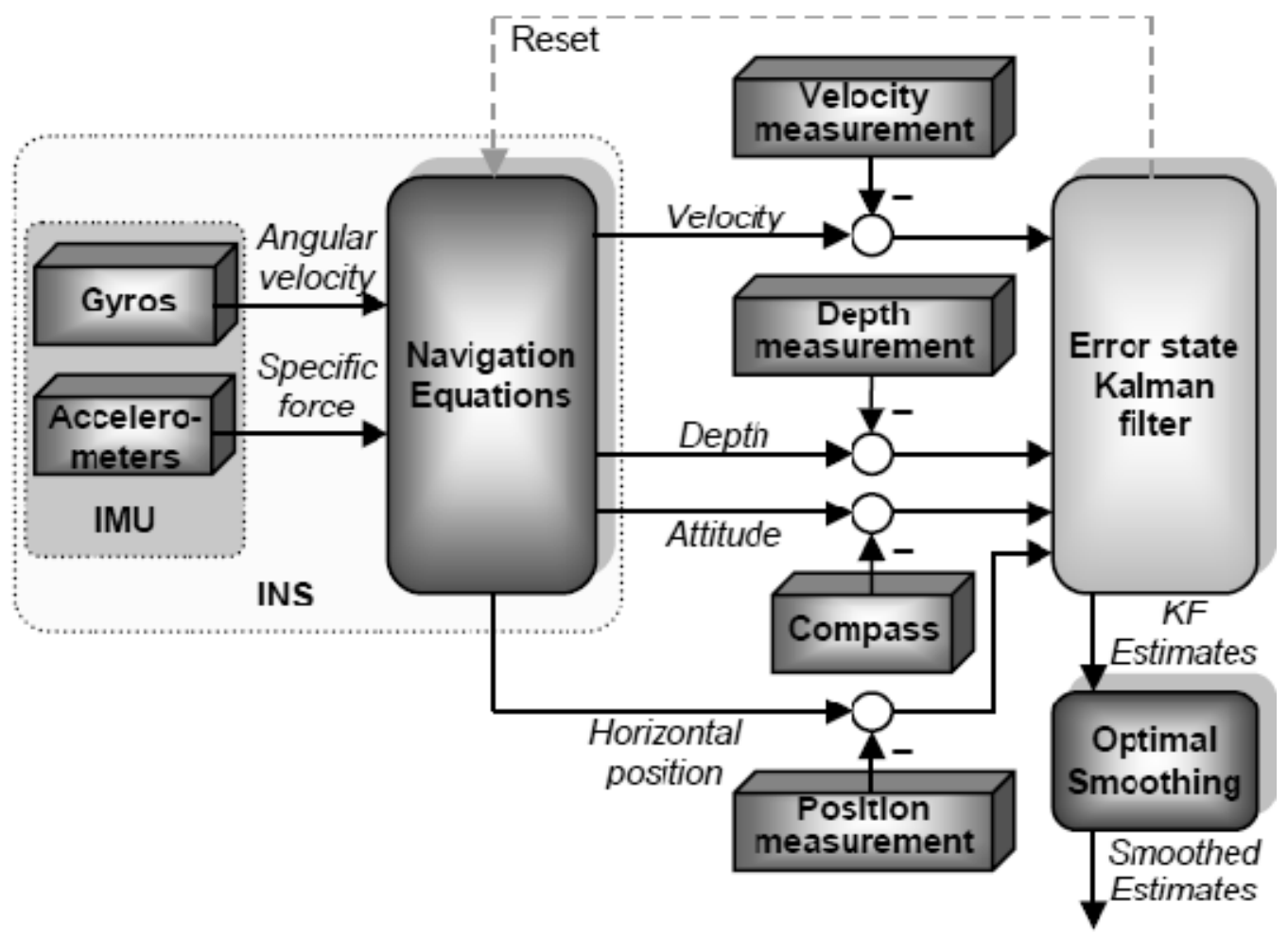

Fig. 2 Aided inertial navigation system

\section{Attitude Computation using Digital MEMS IMU}

For computing the vehicle orientation (roll, pitch, and yaw) angles also called (bank, tilt, and heading) angles using digital MEMS IMU there are two approaches, the first approach is to calculate the Euler angles rates $(\dot{\phi}, \dot{\theta}, \dot{\psi})$ using angular rates obtained from IMU (P, Q, and R) then using one of the integration method to obtain Euler angles using the following equations:

$$
\begin{gathered}
\dot{\varphi}=P+\tan \theta(R \cos \varphi+Q \sin \varphi) \\
\dot{\theta}=Q \cos \varphi-R \sin \varphi \\
\dot{\psi}=(Q \sin \varphi+R \cos \varphi) / \cos \theta \\
\varphi_{\text {new }}=\varphi_{\text {old }}+\dot{\varphi} \Delta t \\
\theta_{\text {new }}=\theta_{\text {old }}+\dot{\theta} \Delta t \\
\psi_{\text {new }}=\psi_{\text {old }}+\dot{\psi} \Delta t
\end{gathered}
$$

The second approach which used in this paper is to calculate Euler angles from quaternion parameters which obtained from IMU using the following equation: 


$$
\begin{aligned}
& \varphi=\tan ^{-1} \frac{2 \times\left(\mathrm{q}_{0} \mathrm{q}_{1}+\mathrm{q}_{2} \mathrm{q}_{3}\right)}{1-2 \times\left(\mathrm{q}_{1}^{2}+\mathrm{q}_{2}^{2}\right)} \\
& \theta=\sin ^{-1}\left(2 \times\left(\mathrm{q}_{0} \mathrm{q}_{2}-\mathrm{q}_{1} \mathrm{q}_{3}\right)\right) \\
& \psi=\tan ^{-1} \frac{2 \times\left(\mathrm{q}_{0} \mathrm{q}_{3}+\mathrm{q}_{1} \mathrm{q}_{2}\right)}{1-2 \times\left(\mathrm{q}_{2}^{2}+\mathrm{q}_{3}^{2}\right)}
\end{aligned}
$$

where the quaternion vector $\left[q_{0} q_{1} q_{2} q_{3}\right]^{T}$ is calculated initially through the alignment process and continuously updated using the rotation rates obtained from the gyroscopes.

The IMU used for computing the orientation with the second approach in this paper is the 6DOF MEMS IMU (ITG3200 gyro + ADXL345 accelerometer) and the results obtained for stationary vehicle shown in Fig. 3, [4].
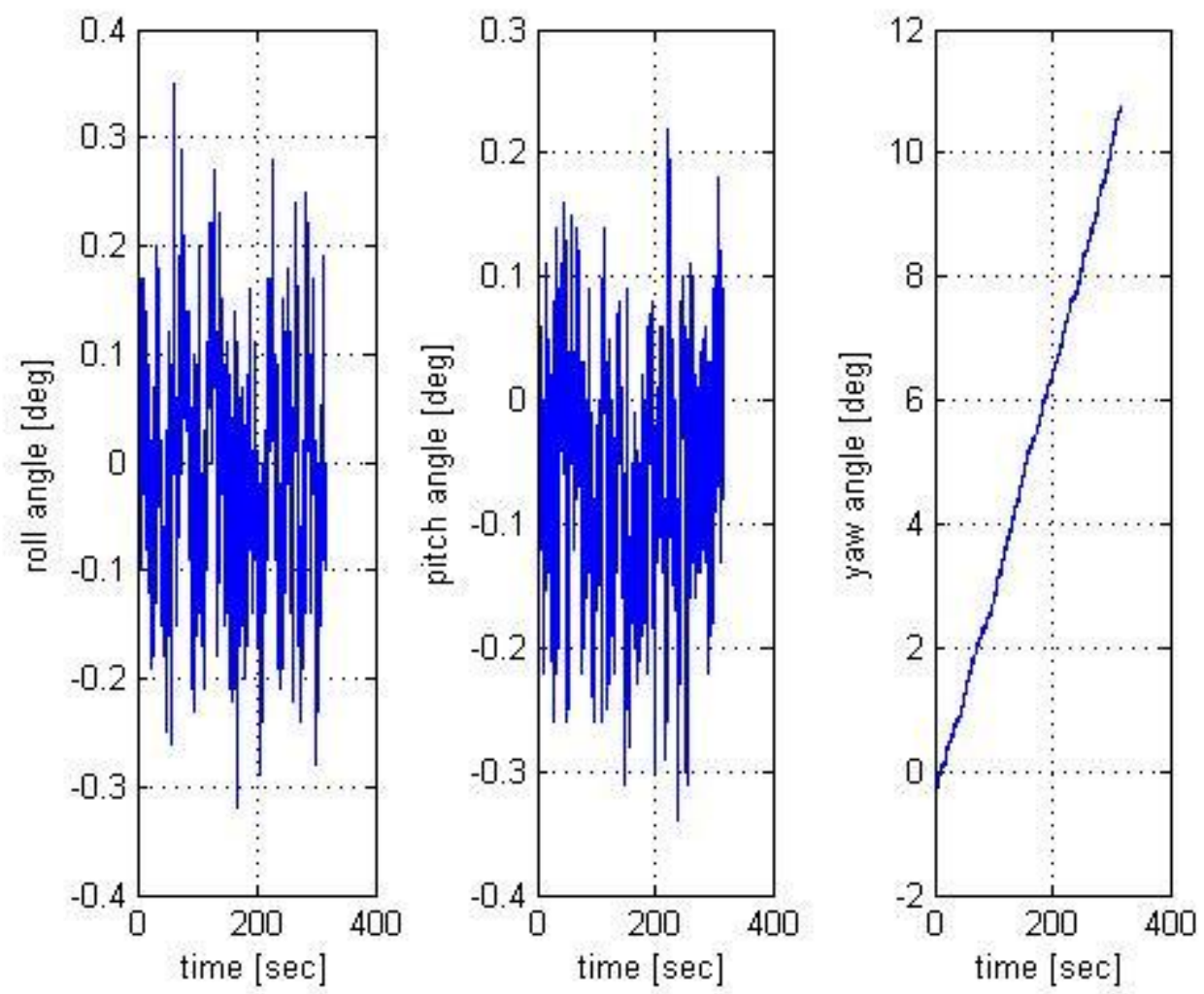

Fig. 3 Stationary vehicle orientation

It can be seen from the previous results that the 6DOF IMU gives high drift in yaw (heading) angle and stable values of roll and pitch angles according to gyro roll and pitch angles drift compensation with connecting the gyroscope (ITG3200) with accelerometer (ADXL345).

\section{Attitude Computation Using Digital MEMS Magnetometers}

A magnetometer is a measuring instrument used to measure the strength and, in some cases, the direction of magnetic fields. Magnetometers are widely used for measuring the Earth's magnetic fields. They are also used militarily to detect submarines. Magnetometers can be used as metal detectors: they can detect only magnetic (ferrous) metals, but can detect such metals at a much larger depth than conventional metal detectors; they are capable of detecting large objects, such as cars, at tens of meters, while a metal detector's range is rarely more than 2 meters. In recent years magnetometers have been used to extent that they can be 
incorporated in integrated circuits at very low cost and are finding increasing use as compasses in consumer devices such as mobile phones and tablet computers.

For yaw (heading) drift compensation magnetometers can be used as they provide a reference vector for yaw orientation of the flying vehicle. In this paper a HMC5883L digital MEMS magnetometer is used for gyro yaw drift compensation [5]. Before using magnetometer, a calibration procedure has to be applied to provide the correct measurements as it is elaborated in the following section.

\subsection{Magnetometer (Compass) Calibration}

Heading can be effectively determined through the use of dual-axis magnetometer and triaxial accelerometer sensors available in an inertial measurement unit (IMU). However, a correctly implemented compass system must compensate for the effect of elevation (pitch) and bank (roll) angle (tilt), as well as calibrate out hard- and soft-iron effects as much as possible. This paper discusses the impact of elevation, bank angle, and hard- and soft-iron effects on heading calculations, and discusses methods that may be employed to counter their impact when using magnetometer data from an IMU. Although the methods address corrections in two dimensions ( $\mathrm{x}-\mathrm{y}$ plane), it is possible, and often necessary, to extend the concepts and functions to three dimensions.

\subsubsection{Elevation and bank angle effect}

Gravity exerts a constant acceleration of $1 \mathrm{~g}$, which may be used to calculate elevation and bank angles. Heading is calculated using the same method as that used in elevation and bank angle, but because gravity cannot be used to calculate changes in heading, the magnetometer data must be used instead. Therefore, to accurately calculate heading, a rotation that removes the bank angle followed by a second rotation that removes elevation or vice versa has to be firstly applied. Once this sequence of rotations is completed the local $x-y$ plane will be realigned with the reference $\mathrm{X}-\mathrm{Y}$ plane, corrections to the magnetometer data will be made, and compensating for hard- and soft-iron effects will be proceeded [6].

\subsubsection{Hard and soft iron distortions}

Distortions of the earth's magnetic field are a result of external magnetic influences generally classified as either a hard- or soft-iron effect. If no distorting effects are present, rotating a magnetometer through a minimum of $360^{\circ}$ and plotting the resulting data as $y$ axis vs. $\mathrm{x}$ axis will result in a circle centered around the point $(0,0)$, as shown in Fig. 4. However, the presence of hard- and/or soft-iron effects may produce a perturbation of the circle as a simple offset from $(0,0)$ in the case of a hard-iron effect, or deform the circle to produce an ellipse in the case of a soft-iron effect. It is also possible that both effects will be exhibited simultaneously as shown in Fig. 5.

It is also important to recognize that effective compensation of hard- and soft-iron distortions is dependent upon the distorting material(s) rotating/moving with the sensor. An example would be mounting the sensor in an aircraft; any materials that are part of the aircraft that exhibit a distorting effect would move as the aircraft and mounted sensor move, and it would generally be possible to compensate for the associated hard- and soft-iron effects. In contrast, it is much more difficult-if not impossible-to compensate for distorting effects exhibited by material external to the aircraft/sensor platform. Thus, it is important to understand not only how compensation may be applied, but also to recognize those conditions under which effective compensation techniques are not possible. Non-calibrated magnetometer gives wrong heading readings as shown in Fig. 6. 


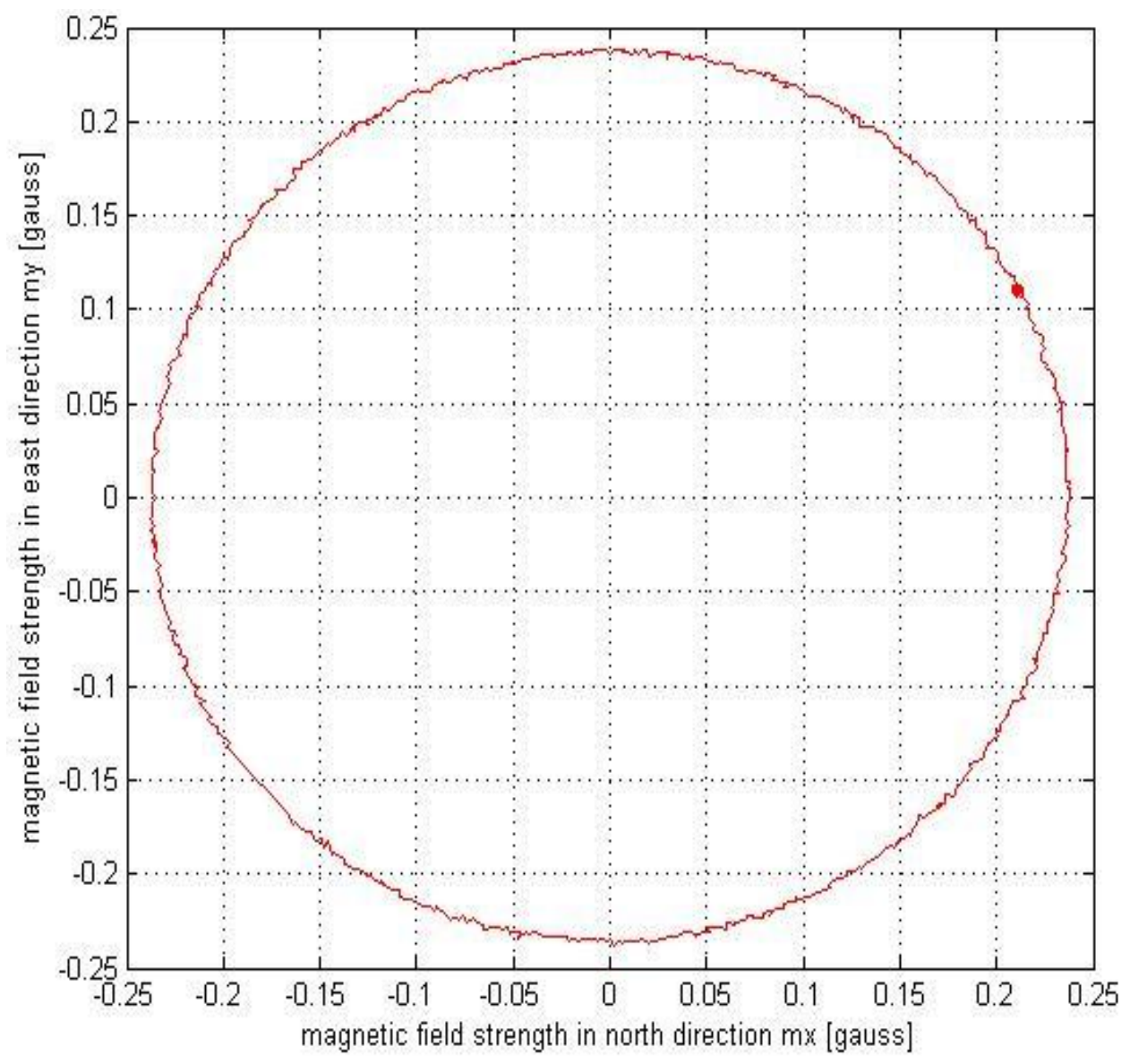

Fig. 4 A graph of ideal distortion-free magnetometer data. (Note that output is centered around $(0,0)$ and circular in shape)

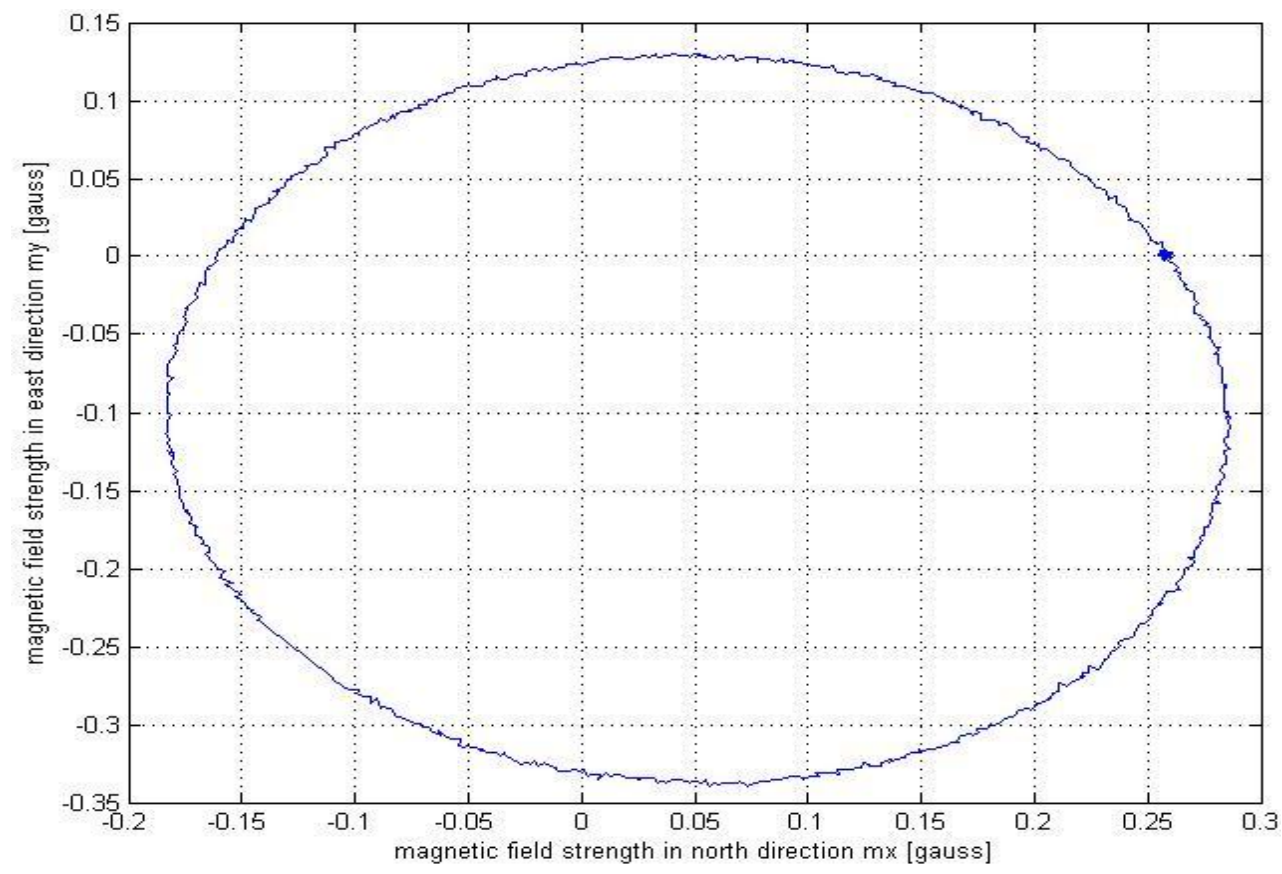

Fig. 5 A graph of magnetometer with both hard and soft iron distortion 


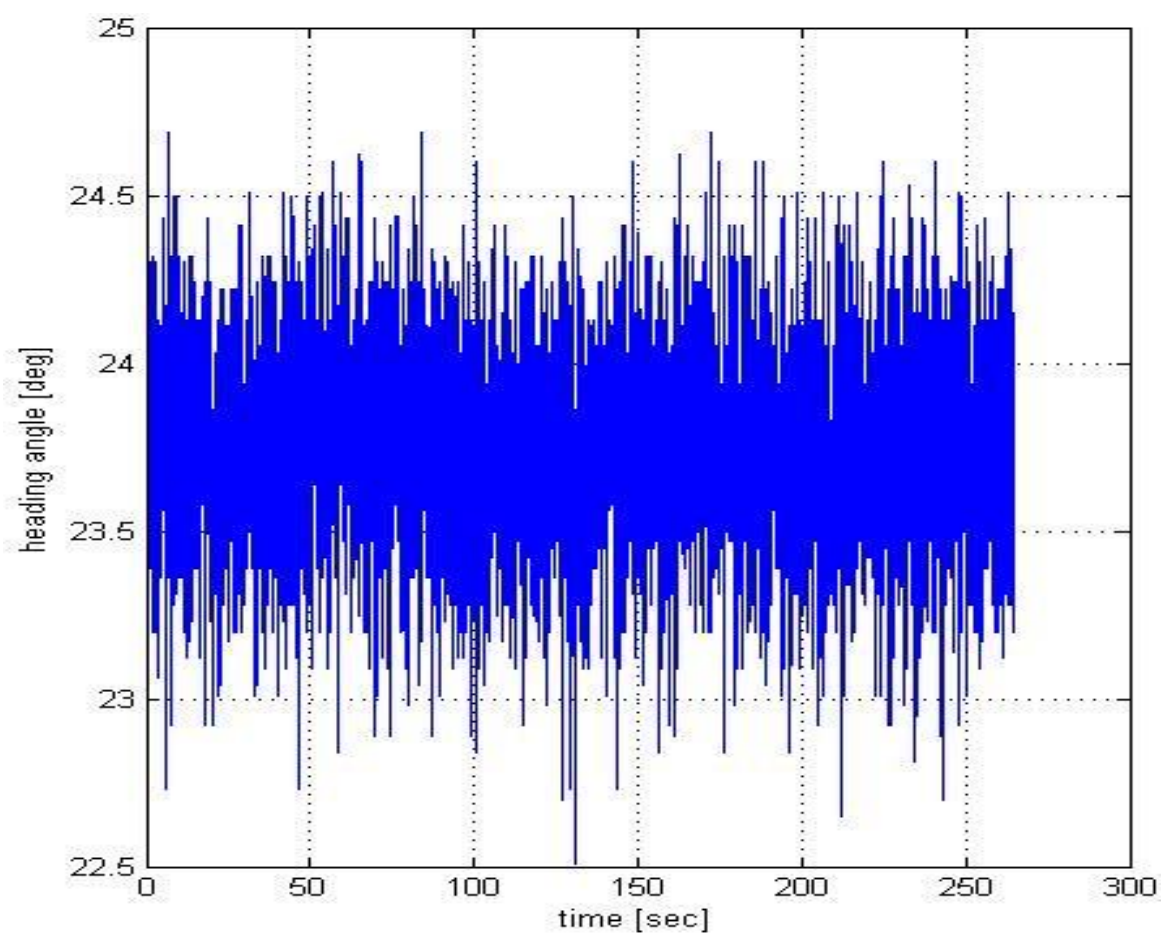

Fig. 6 Heading angle of non-calibrated magnetometer for a vehicle points to north direction

\subsection{Hard Iron Distortions Compensation}

Hard-iron distortion is produced by materials that exhibit a constant, additive field to the earth's magnetic field, thereby generating a constant additive value to the output of each of the magnetometer axes. A speaker magnet, for example, will produce a hard-iron distortion. As long as the orientation and position of the magnet relative to the sensor is constant the field and associated offsets will also be constant. A hard-iron distortion can be visibly identified by an offset of the origin of the ideal circle from $(0,0)$ as shown in Fig. 5. Compensating for hard-iron distortion is straightforward, accomplished by determining the $\mathrm{x}$ and $\mathrm{y}$ offsets and then applying these constants directly to the data. It is important to note that tilt compensation must be applied prior to determining hard-iron corrections. Hard-iron corrections are typically determined by rotating the sensor through a minimum of $360^{\circ}$, then determining the distance from $(0,0)$ to the center of the circle by identifying the average of the maximum and minimum values for each of the axes, as shown in equations 15 and 16, respectively. The table used in magnetometer calibration shown in Fig. 7, [7].

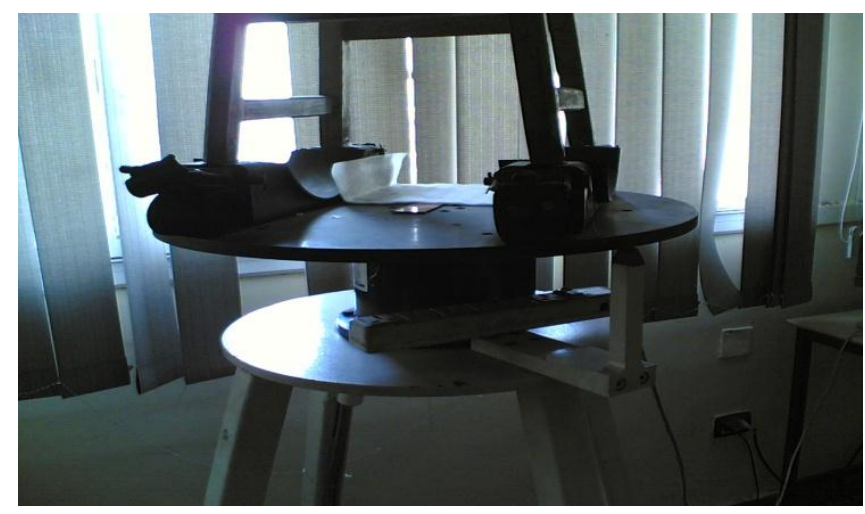

Fig. 7 Turn table used in magnetometer calibration 


$$
\begin{aligned}
& \alpha=\frac{x_{\text {max }}+x_{\text {min }}}{2} \\
& \beta=\frac{y_{\text {max }}+y_{\text {min }}}{2}
\end{aligned}
$$

where:

$$
\begin{array}{ll}
\alpha & \mathrm{X} \text { axis offset } \\
x_{\max } & \text { Maximum x value } \\
x_{\min } & \text { Minimum x value } \\
\beta & \text { Y axis offset } \\
y_{\max } & \text { Maximum y value } \\
y_{\min } & \text { Minimum y value }
\end{array}
$$

These offsets are then subtracted from the raw $\mathrm{x}$ and $\mathrm{y}$ magnetometer data, thus largely eliminating the hard-iron distortion. Hard-iron effects are constant regardless of orientation or position of the sensing platform. These constant offsets can be stored once calculated and subtracted from the raw magnetometer data. A magnetometer with hard iron compensation and soft iron distortion is shown in Fig. 8. Heading angle after hard iron compensation and with soft iron distortion shown in Fig. 9.

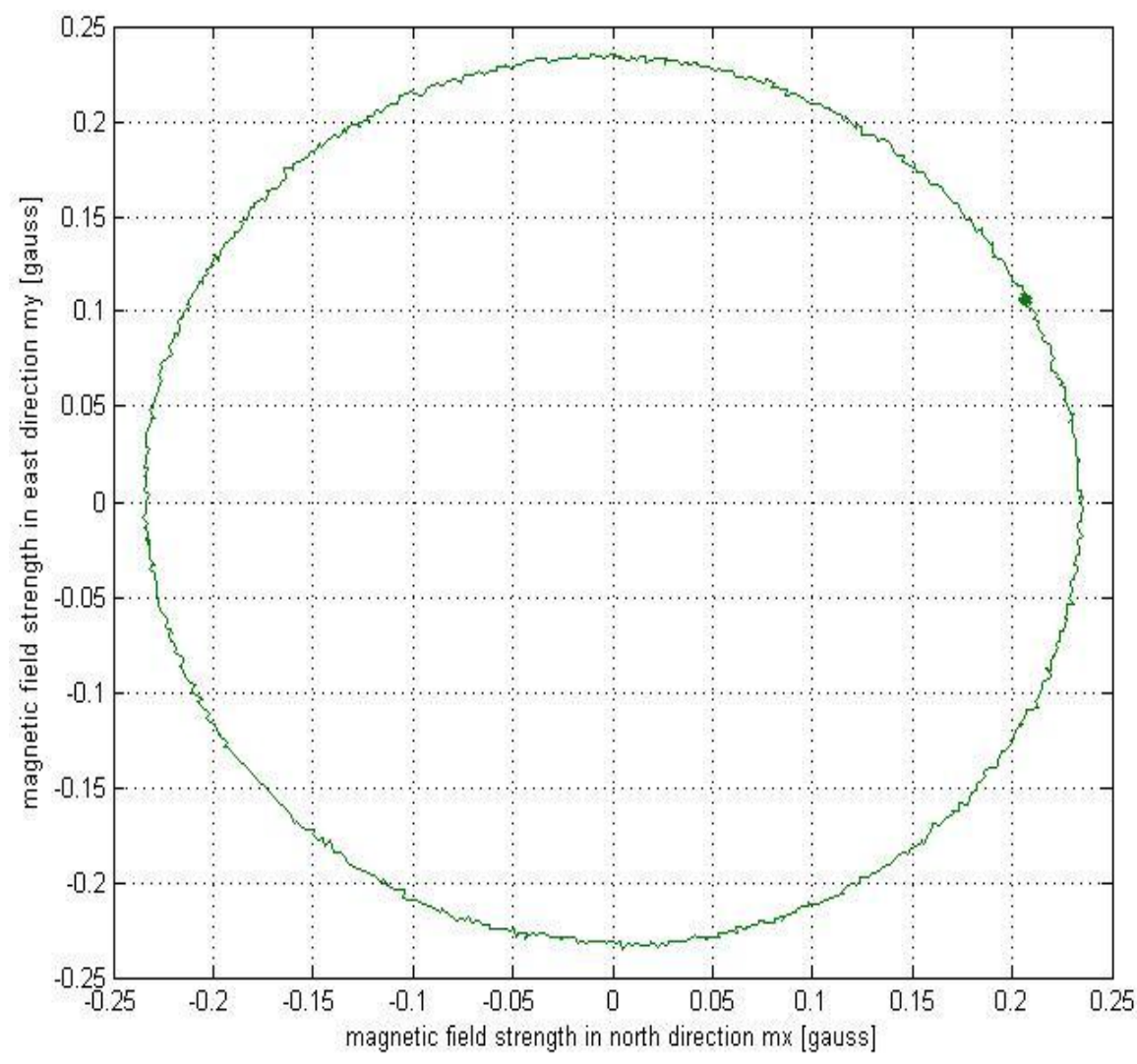

Fig. 8 A graph of magnetometer with hard iron distortion compensation and soft iron distortion 


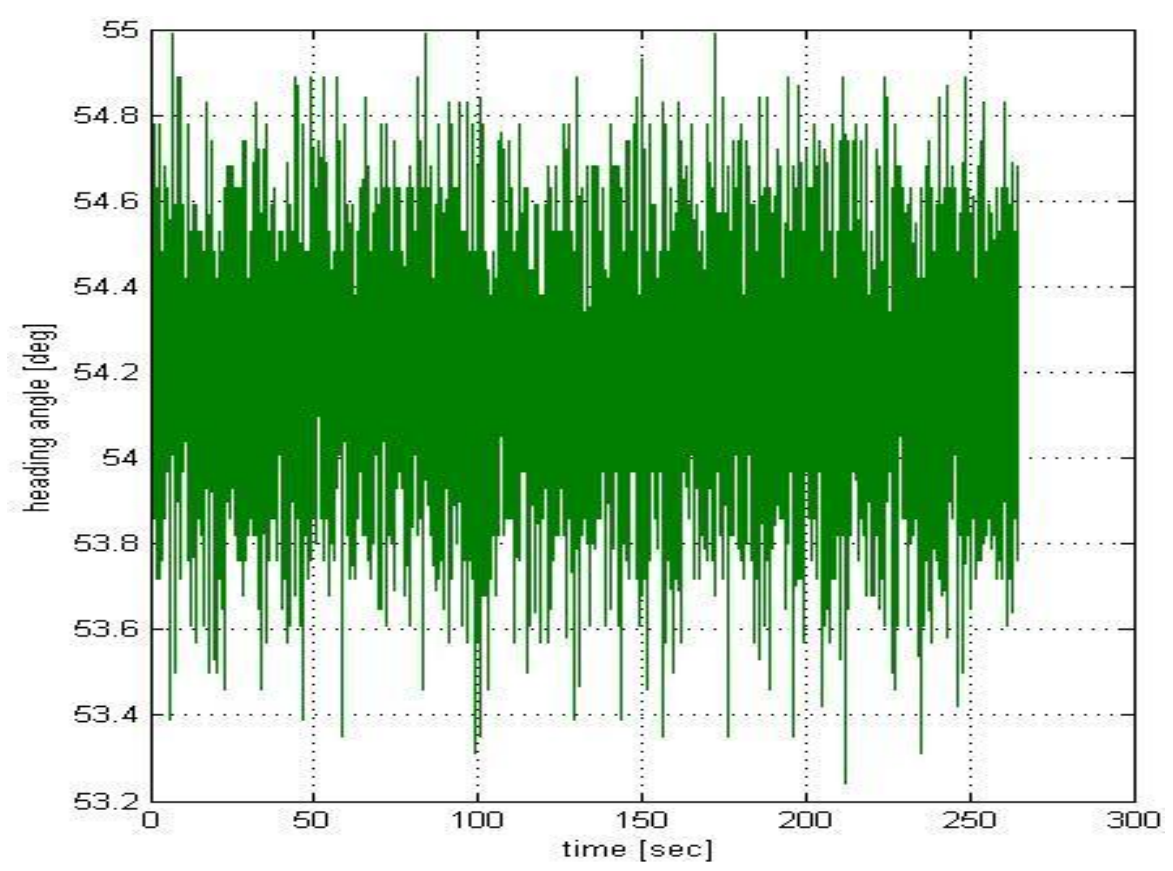

Fig. 9 Heading angle of magnetometer with hard iron compensation and soft iron distortion for a vehicle points to north direction

\subsection{Soft Iron Distortions Compensation}

Unlike hard-iron distortion where the magnetic field is additive to the earth's field, soft-iron distortion is the result of material that influences, or distorts, a magnetic field but does not necessarily generate a magnetic field itself, and is therefore not additive. Iron and nickel, for example, will generate a soft-iron distortion. While hard-iron distortion is constant regardless of orientation, the distortion produced by soft-iron materials is dependent upon the orientation of the material relative to the sensor and the magnetic field. Thus, soft-iron distortion cannot be compensated with a simple constant; instead, a more complicated procedure is required. To simplify the following discussion we assume that all tilt effects and hard-iron distortions are either not presents in the application environment, or that tilt and hard-iron compensation methods have previously been applied. Furthermore, if tilt and hard-iron effects are present, compensation for these distortions must be applied prior to correcting for soft-iron distortions. As such, it is safe to assume that the origin of the ellipse is at $(0,0)$ as shown in Fig. 10, and is exhibiting a rotation of $\theta$ degrees from the $\mathrm{X}$ axis.

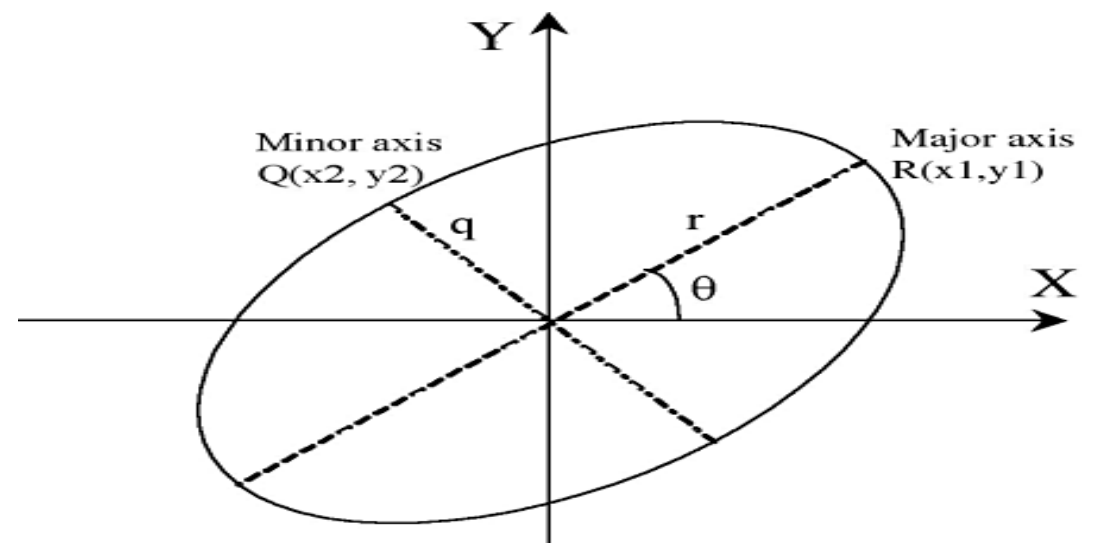

Fig. 10 An ellipse generated as a result of soft-iron distortion centered at $(0,0)$ with rotation $\theta$ 
Identifying $\theta$ in Fig. 10 is accomplished by using equation (12) to calculate the magnitude of the line segment $r$, followed by equation (13) to determine $\theta$ :

$$
\begin{gathered}
r=\sqrt{x_{1}^{2}+y_{1}^{2}} \\
\theta=a \sin \left(\frac{y_{1}}{r}\right)
\end{gathered}
$$

Once $\theta$ has been identified, the rotation matrix given in equation (14) is applied to the vector of magnetometer $\mathrm{x}$ and $\mathrm{y}$ values, $\mathrm{v}$, using equation (15) [8].

$$
\begin{gathered}
R=\left[\begin{array}{cc}
\cos \theta & \sin \theta \\
-\sin \theta & \cos \theta
\end{array}\right] \\
v_{1}=R v
\end{gathered}
$$

After the rotation, the major axis of the ellipse will be aligned with the reference frame $\mathrm{X}$ axis and the minor axis will be aligned with the Y axis, as shown in Fig. 11.

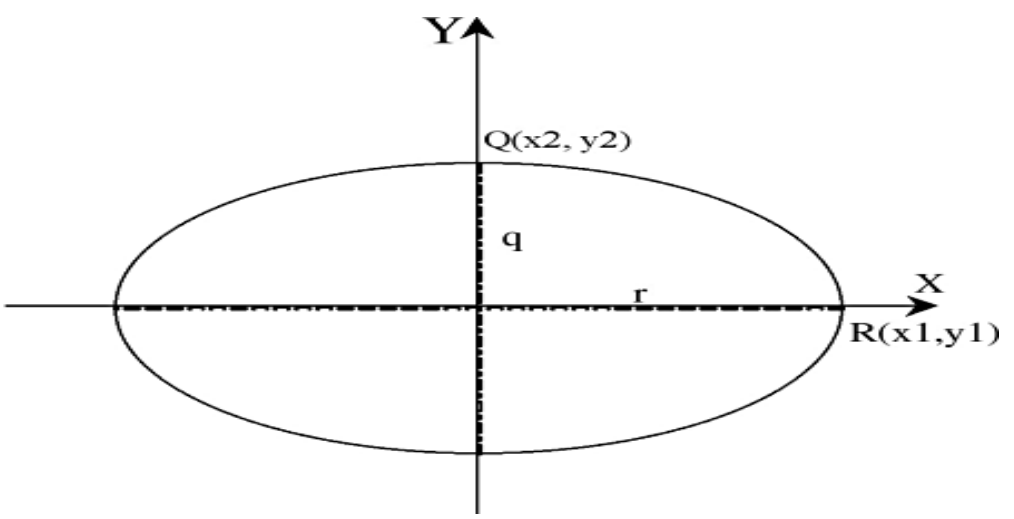

Fig. 11 Alignment of the ellipse's major and minor axes with the coordinate system's $x$ and $y$ axes, respectively, following the rotation

Following the rotation, we can now properly scale the major axis such that the ellipse is converted to an approximate circle. The scale factor, $\sigma$, is determined using equation (16), and is the ratio of the length of the major axis to that of the minor axis. Each magnetometer $\mathrm{x}$ value is then divided by this scale factor to produce the desired circle as shown in equations $(17,18)$.

$$
\begin{gathered}
\sigma=\frac{q}{r} \\
m_{x}=\left(m_{x_{1}} / \sigma\right) \cos \theta+\left(m_{y_{1}} / \sigma\right) \sin \theta \\
m_{y}=\left(-m_{x_{1}} / \sigma\right) \sin \theta+\left(m_{y_{1}} / \sigma\right) \cos \theta
\end{gathered}
$$

Once scaling is completed a final rotation must be made to rotate the data back to their original position, thus compensating for the soft-iron distortion. A magnetometer with hard and soft iron compensations is shown in Fig. 12. Heading angle after hard and soft iron compensation distortion shown in Fig. 13. Magnetometer measurement before and after calibration is shown in Fig. 14. A comparison between magnetometer heading measurement before calibration and after hard and soft iron calibrations is shown in Fig. 15. 


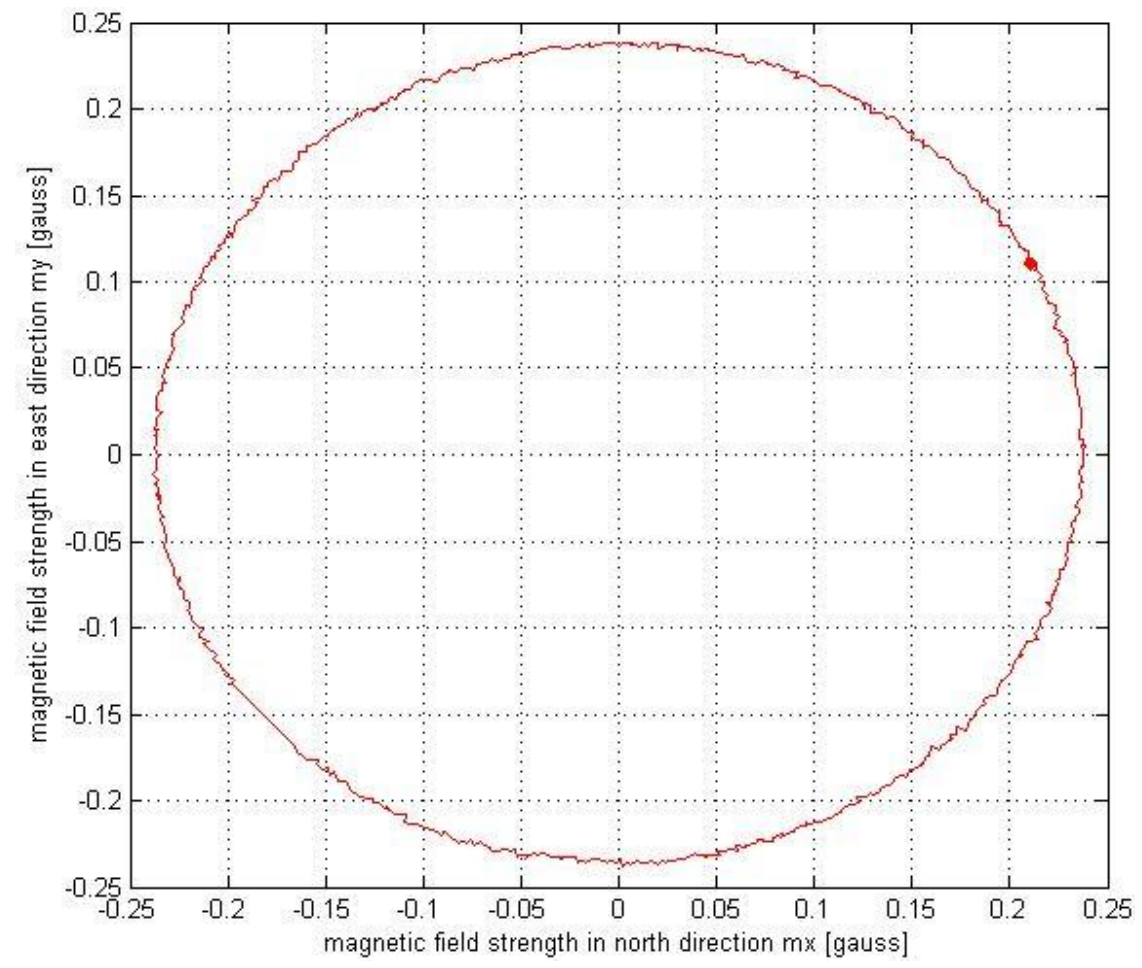

Fig. 12 A graph of magnetometer with hard and soft iron distortion compensation

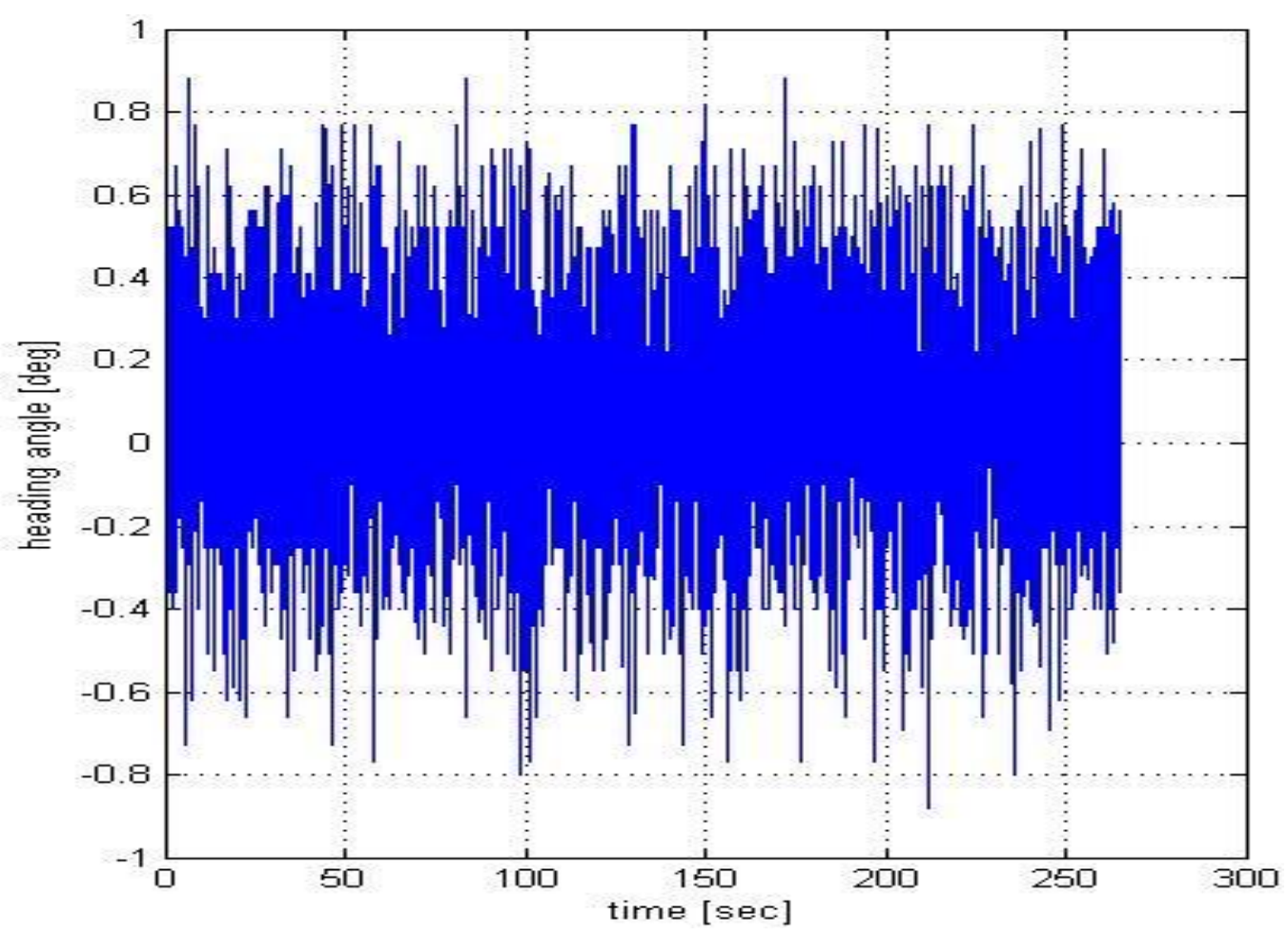

Fig. 13 Heading angle of magnetometer with hard and soft iron distortion compensation for a vehicle points to north direction 


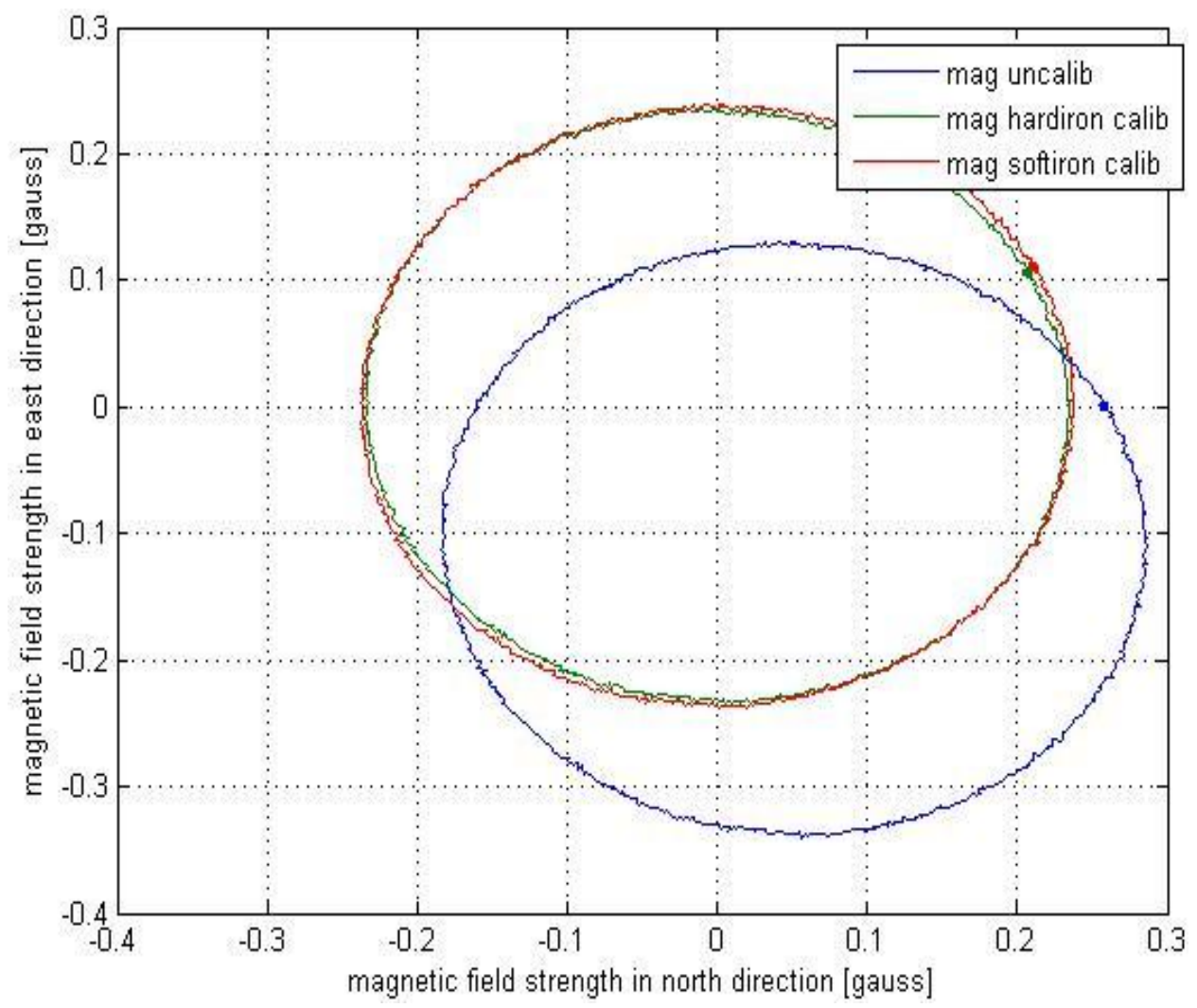

Fig. 14 A graph of magnetometer before and after calibration

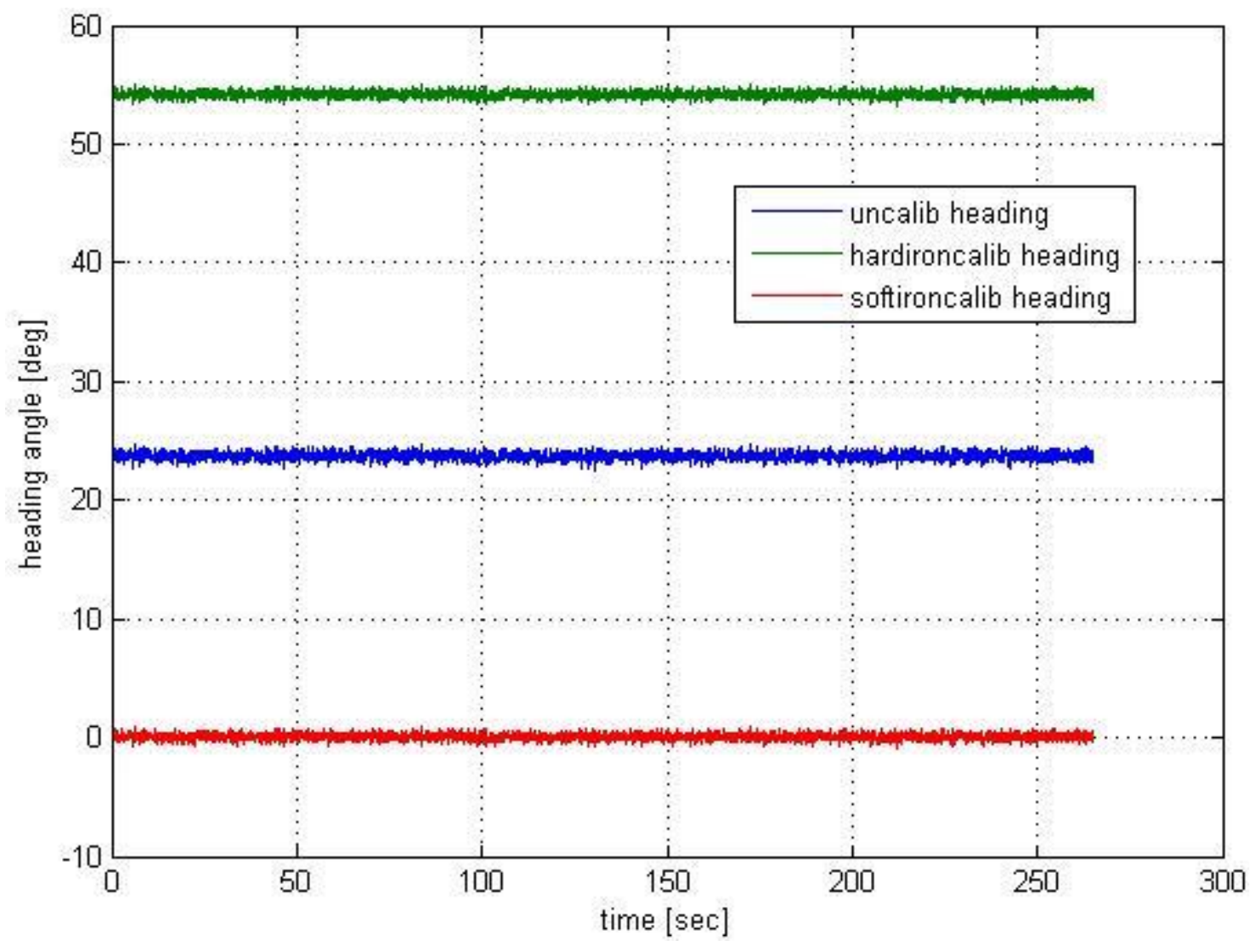

Fig. 15 Heading angle of magnetometer before calibration and after hard and soft iron distortion compensation for a vehicle points to north direction 
After the previous steps, the magnetometer is well calibrated and can compensate the known errors caused by hard and soft iron effects as described earlier. However, if the magnetometer is disturbed by an external source such as iron a sudden and temporary error will occur and incorrect heading measurements is provided as shown in Fig. 16. For this reason, an optimal estimator such as Kalman filter (KF) is used to overcome these types of errors and provide smooth attitude measurements in addition as described in the following section.

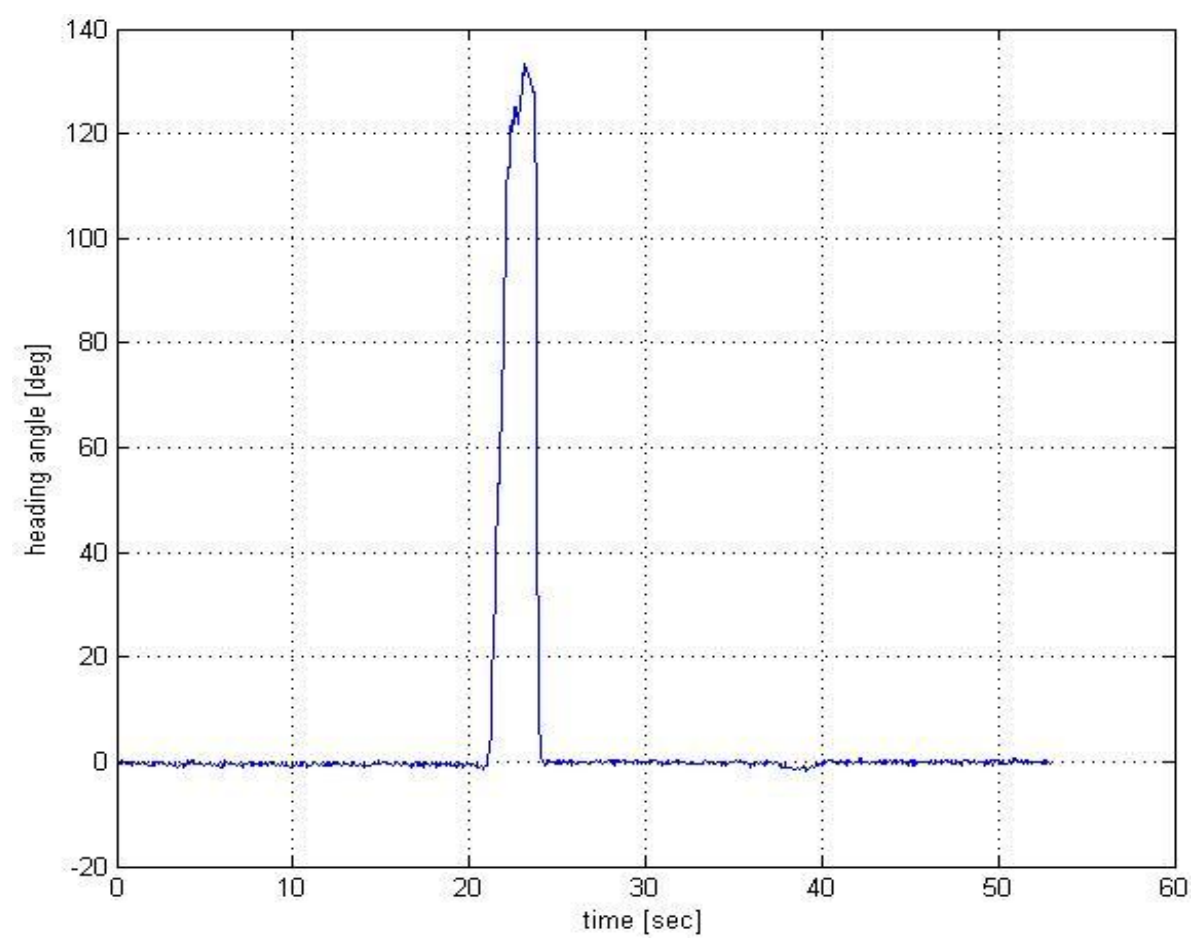

Fig. 16 Heading angle of magnetometer disturbed by iron source for a vehicle points to north direction

\section{Kalman Filter (KF)}

KF based estimation and tracking systems are widely used for aviation and military applications. The KF designer faces several challenging tasks such as designing the dynamic model design and a correct description of the noise matrices, which are related to the measurements and process.

KF estimates its process states using a form of feedback control by estimating the process state and then obtaining feedback in the form of noisy measurements. As such, the KF equations are categorized into two sets described as time update equations and measurement update equations. The time update equations are responsible for projecting forward in time the current state and error covariance estimates to obtain the a priori estimates for the next time step. The measurement update equations are responsible for the feedback by incorporating a new measurement into the a priori estimate to obtain an improved a posteriori estimate.

$\mathrm{KF}$ equations for time and measurement updates can be written as [9]: 


$$
\begin{gathered}
X_{k+1}^{-}=\Phi_{k, k+1} X_{k}^{+}+G w_{k}, \\
P_{k+1}^{-}=\Phi_{k, k+1} P_{k}^{+} \Phi_{k, k+1}^{T}+Q, \\
K_{k}=P_{k}^{-} H^{T}\left(H P_{k}^{-} H^{T}+R\right)^{-1}, \\
X_{k}^{+}=X_{k}^{-}+K_{k}\left(Z_{k}-H X_{k}^{-}\right), \\
P_{k}=\left(I-K_{k} H\right) P_{k}^{-}
\end{gathered}
$$

where $X$ represents the state vector, $\Phi$ represents the transition matrix, $P$ is the error covariance matrix, $R$ and $Q$ are the measurement and process noise covariance matrices, $K$ is the Kalman gain, $Z$ is update measurements, and $H$ is the observation model, which maps the true state space into the observed space. The negative sign (-) denotes prediction based on model dynamics only, whereas the positive sign $(+)$ denotes correction accounting for the measurements.

The first task during the measurement update is to compute the Kalman gain, K. The next step is to actually measure the designated state data input, and then to generate an a posteriori state estimate by incorporating the measurement as in Equations (19, 20, 21, 22, 23). The final step is to obtain an a posteriori error covariance estimate. After each state prediction and measurement update pair, the process is repeated with the previous a posteriori estimates used to project or predict the new a priori estimates. This process is illustrated in Figs. 17 and 18.

In the actual implementation of the filter, the measurement noise covariance $\mathrm{R}$ is usually measured prior to operation of the filter. The determination of the process noise covariance $\mathrm{Q}$ is generally more difficult because practically there is no direct observation of the process during estimation.

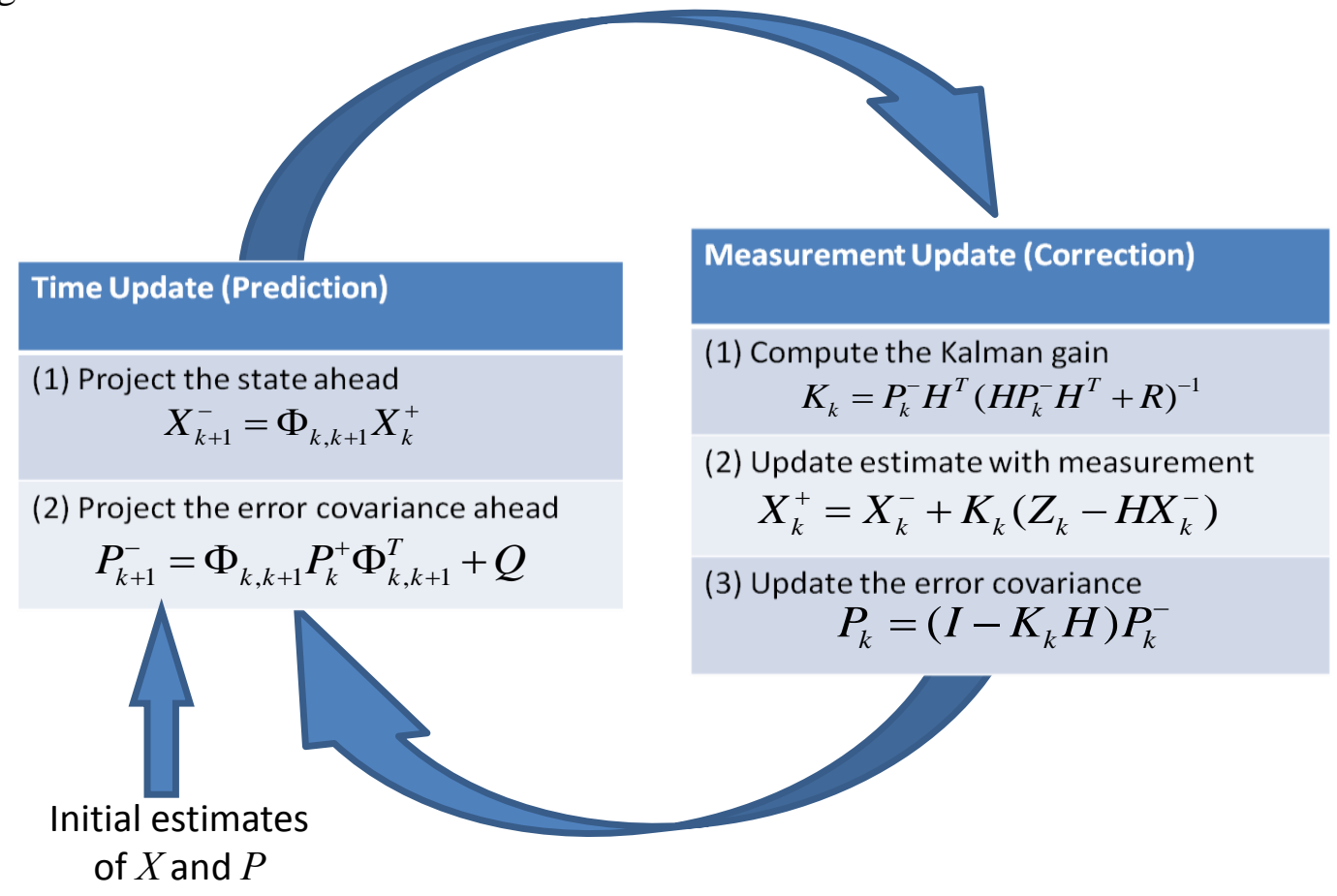

Fig. 17 Kalman filter process 


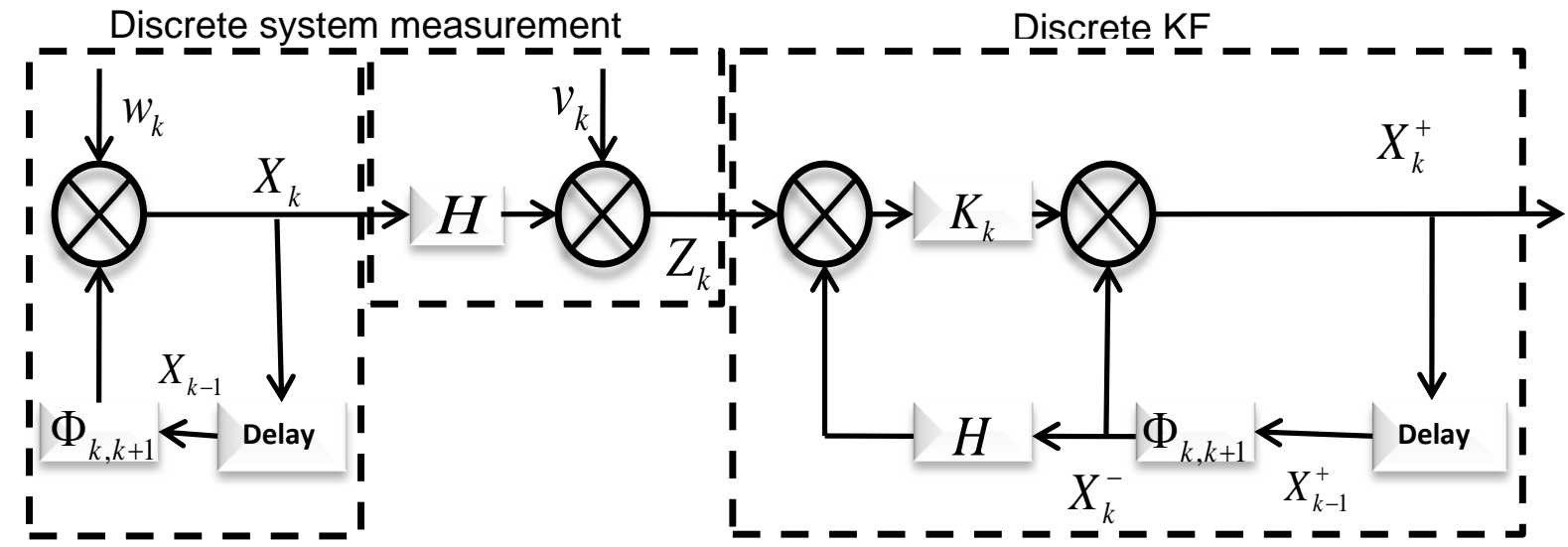

Fig. 18 Block diagram of a system, a measurement model, and a discrete Kalman Filter

A simple Kalman filter is used for smoothing the roll, pitch, and yaw measurements. The Kalman filter model parameters used are the following:

$$
\begin{gathered}
\Phi=[1] \\
H=[1] \\
\mathrm{Q}=[0.0001] \\
\mathrm{R}=[1] \\
\mathrm{P}=[1]
\end{gathered}
$$

The roll, pitch, and yaw measurements after using Kalman filter is illustrated in Fig. 19 and the standard deviation shown in Table 1.

Heading angle measured by magnetometer before and after disturbance rejection using Kalman filter is shown in Fig. 20.
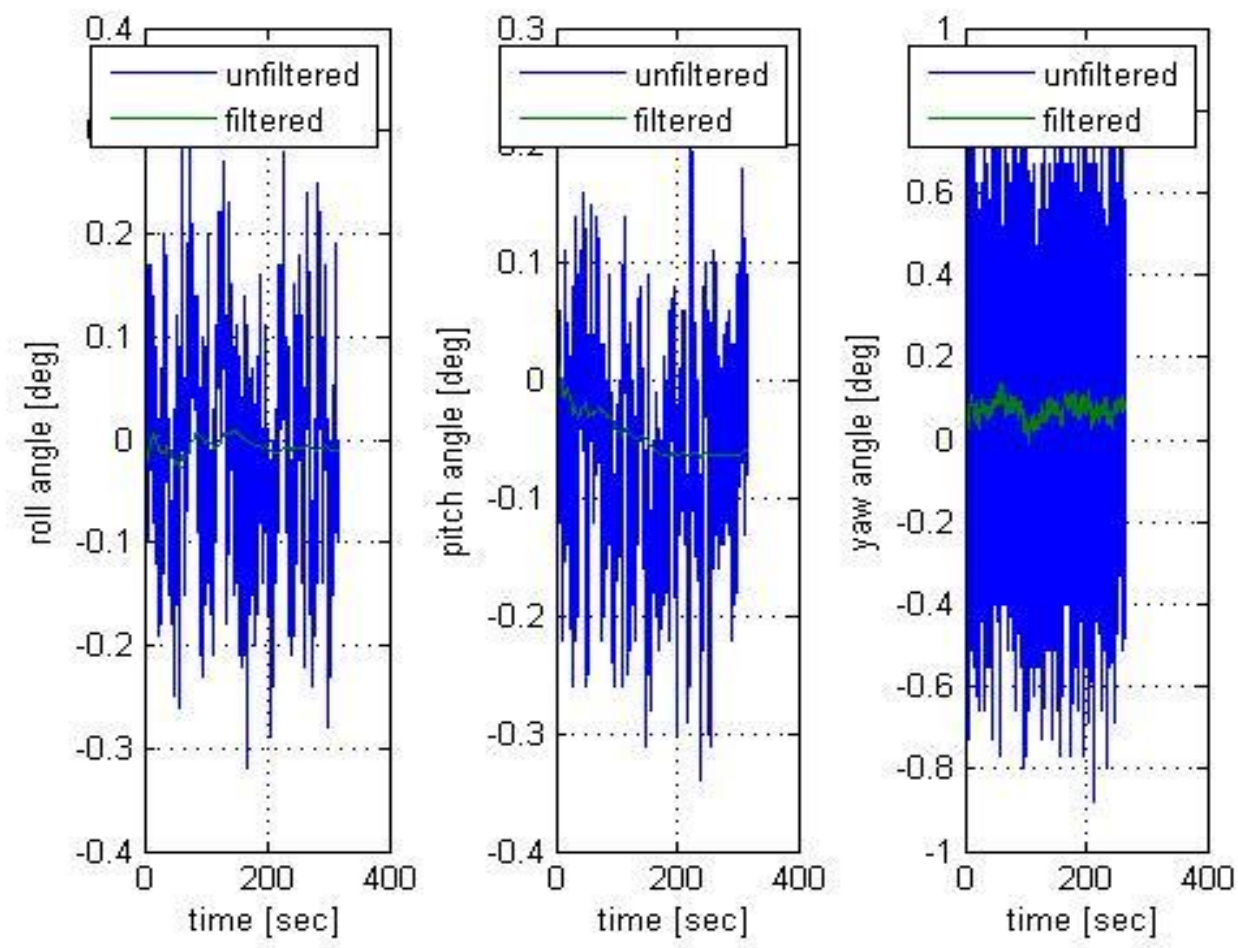

Fig. 19 Roll, pitch, and yaw angles before and after using Kalman filter 
Table 1: Unfiltered and filtered orientation standard deviation

\begin{tabular}{c|c|c|c}
\hline \hline & Roll [deg] & Pitch [deg] & Yaw [deg] \\
\hline Unfiltered & 0.1085 & 0.08708 & 0.2666 \\
\hline Filtered & 0.007451 & 0.01956 & 0.02274 \\
\hline \hline
\end{tabular}

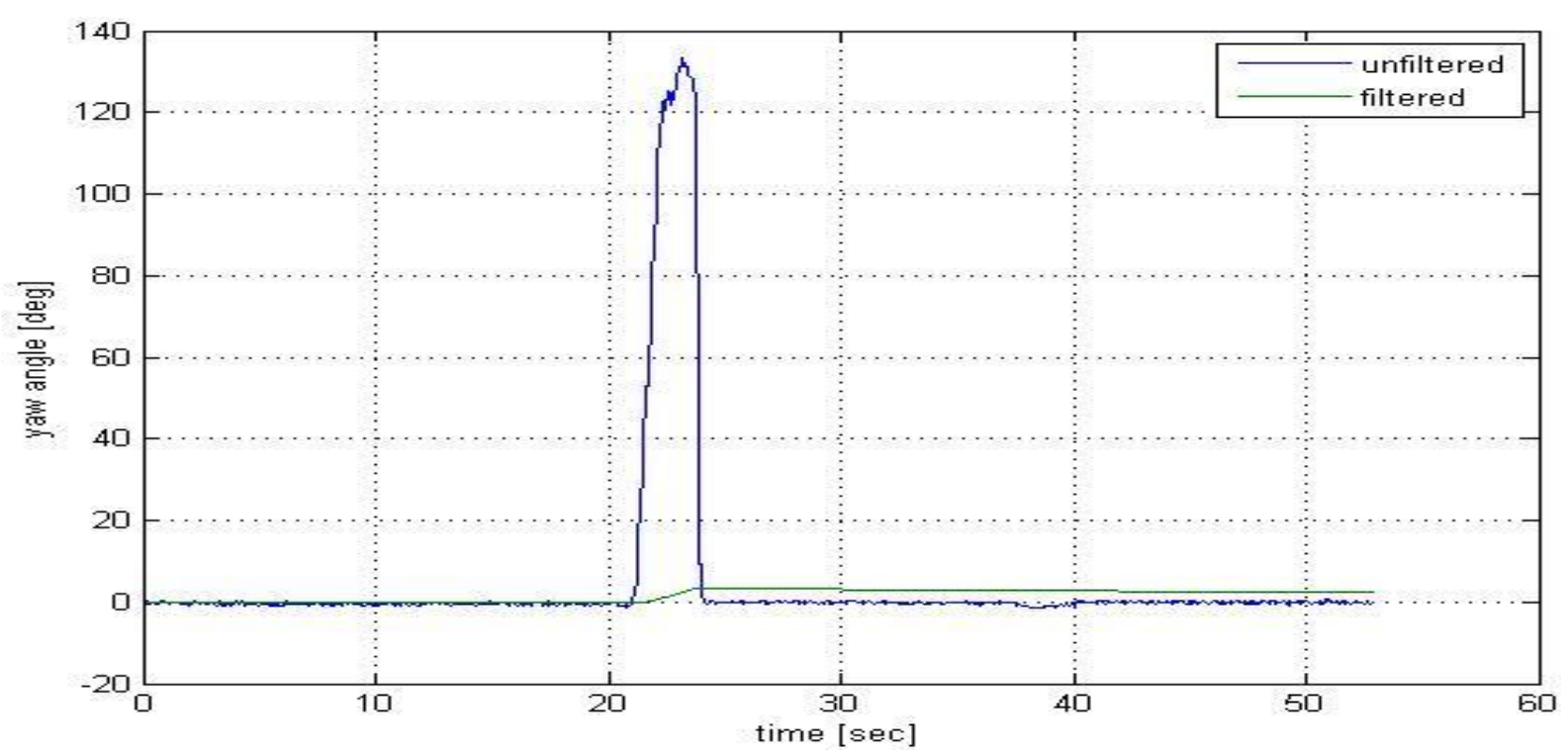

Fig. 20 Heading angle measured by magnetometer before and after disturbance rejection using Kalman filter

\section{Conclusion}

For military applications, it is not accepted to use GNSS as a sole mean for navigation as it is susceptible to jamming and signal interference. Measuring orientation from gyroscope only suffers from high drift with time. For roll and pitch drift compensation an accelerometer aided to gyroscope and for yaw drift compensation a magnetometer aided to gyroscope. For accurate orientation measurement the sensors (gyros, accelerometers and magnetometers) should be calibrated.

\section{References}

[1] Rolf Christensen, NikolajFogh, "Inertial Navigation System", Aalborg University, Department of Electronic Systems, Denmark (2008).

[2] Sherry H. Stovall, "Basic Inertial Navigation", naval air warfare center weapons division, System integration branch, 1997.

[3] Kenneth Gade, "Introduction to Inertial Navigation", forsvarets forsknings institute, 2004.

[4] "IMU Digital Combo Board 6 Degrees of Freedom ITG3200/ADXL345", from https://www.sparkfun.com/products/10121.

[5] "Triple Axis Magnetometer Breakout - HMC5883L", from https://www.sparkfun.com/products/10530.

[6] Konvalin, C.J., June 28, 2008, "Calculating Bank, Elevation and Heading." (PDF), retrieved: August 4, 2008, from http://memsense.com.

[7] Danaher Motion, "Setup andReference Guide", Kollmorgen, a Danaher Corporation, United States of America, 2002. 
[8] Jack B. Kuipers, "Quaternions and Rotation Sequences, a Primer with Applications to Orbits, Aerospace, and Virtual Reality”, Princeton University Press, Princeton, 2002.

[9] Gary Bishop, Greg Welch, "An Introduction to the Kalman Filter", Department of Computer Science, University of North Carolina at Chapel Hill, 2006. 